\title{
Polar wind outflow model: Saturn results
}

\author{
A. Glocer, ${ }^{1}$ T. I. Gombosi, ${ }^{1}$ G. Toth, ${ }^{1}$ K. C. Hansen, ${ }^{1}$ A. J. Ridley, ${ }^{1}$ and A. Nagy ${ }^{1}$ \\ Received 29 March 2006; revised 14 August 2006; accepted 24 October 2006; published 20 January 2007.
}

[1] The Saturnian system's configuration and dynamics are to a large extent controlled by the planet's rapid rotation and the plasma in the magnetosphere. Therefore characterizing the relative importance of the various plasma sources is crucial to understanding Saturn's magnetosphere. Most research in this area focuses on the addition of mass from the icy satellites, the rings, and Titan, while comparatively little attention has been paid to the ionospheric source. We investigate the ionospheric source at high latitude using multifluid numerical simulations of Saturn's polar wind and find that the magnitude of the particle source rate out of the polar cap is between $2.1 \times 10^{26}$ and $7.5 \times 10^{27} \mathrm{~s}^{-1}$. Our multifluid simulations are carried out using the Polar Wind Outflow Model (PWOM). This new model is capable of calculating the polar wind at Earth and Saturn by solving the gyrotropic transport equations. The polar wind at Saturn is modeled from below the peak ionospheric density to an altitude of one Saturn radius, yielding fluxes for $\mathrm{H}_{3}^{+}, \mathrm{H}^{+}$, and electrons. Because the neutral temperature is ill constrained, we calculate source rates for various Saturnian atmospheric profiles corresponding to neutral temperatures of 420,600,800, 1000, $1500 \mathrm{~K}$. We compare the results with those calculated from other models and measurements where appropriate.

Citation: Glocer, A., T. I. Gombosi, G. Toth, K. C. Hansen, A. J. Ridley, and A. Nagy (2007), Polar wind outflow model: Saturn results, J. Geophys. Res., 112, A01304, doi:10.1029/2006JA011755.

\section{Introduction}

[2] The Saturnian system has been explored and understood using a variety of methods including Earth based observation, in situ measurements, and numerical simulations. Cassini's arrival at Saturn in 2004 has dramatically increased the amount of new data about Saturn's magnetosphere. By using numerical simulations, we can improve our understanding of these new measurements. In particular, we focus on the ionosphere's relative importance as a sources of magnetospheric plasma.

[3] In order to understand the importance of Saturn's various plasma sources, we draw an analogy to the environment at Earth. The Earth's ionosphere is an important plasma source for the magnetosphere. Sharp et al. [1985] examine ISEE 1 and SCATHA mass spectrometer data and determine that the ionosphere is an important or dominant source of plasma for the inner magnetosphere. Furthermore, the significance of an ionospheric source led to speculation on the existence of a geopause [Moore and Delcourt, 1995]. A recent paper by Huddleston et al. [2005] examines data from the Dynamics Explorer, and Polar spacecrafts, and argues that the ionosphere is a sufficient source for magnetospheric plasma. However, the ionosphere is not the only controller of magnetospheric plasma. Solar wind parameters

\footnotetext{
${ }^{1}$ Center for Space Environment Modeling, University of Michigan, Ann Arbor, Michigan, USA.

Copyright 2007 by the American Geophysical Union. 0148-0227/07/2006JA011755
}

are found to be highly correlated to plasma sheet parameters, and the occurrence of the super dense plasma sheet is found to be related to increased density in the solar wind [Borovsky et al., 1997; Borovsky et al., 1998]. Winglee [2000] uses multifluid simulations that include both the ionospheric and solar wind source and finds that the relative importance is highly variable. These studies indicate that the dominant source is highly dependent on geophysical conditions. However, the ionosphere is clearly an important, source of the magnetospheric plasma at the Earth. Because the ionosphere plays such an important role at Earth, there is reason to believe it may be a factor at Saturn.

[4] Most previous studies of Saturn's magnetosphere have focused on nonionospheric sources. Richardson and Jurac [2004] use a self-consistent model of plasma and neutrals to estimate the source rate of water products into the magnetosphere. Their model, described fully by Jurac and Richardson [2005], assumes that 95 percent of the neutral source is water and 5 percent is hydrogen. The model solves a diffusion equation for the transport of $\mathrm{H}^{+}$, $\mathrm{H}_{2}^{+}, \mathrm{O}^{+}, \mathrm{OH}^{+}, \mathrm{H}_{2} \mathrm{O}^{+}$, and $\mathrm{O}^{++}$, and the neutral distribution is determined using a Monte Carlo method. They constrain their model with Voyager and Hubble Space Telescope data, and estimate a source of about $10^{28} \mathrm{H}_{2} \mathrm{O} / \mathrm{s}$ coming from the area around Enceladus. Gombosi and Hansen [2005] show that this source can be very significant in determining magnetospheric behavior. By applying a plasma source of $10^{28} \mathrm{ions} / \mathrm{s}$, which assumes that all of the neutral particles produced in the inner magnetosphere are ionized, to a global three-dimensional (3-D) magnetohydrodynamic simulation, 
they demonstrate that quasiperiodic behavior of the magnetosphere can result.

[5] A mass source originating from Enceladus has recently been measured by numerous instruments on the Cassini spacecraft. The Cassini Magnetometer detected the interaction of Saturn's plasma environment with an atmospheric plume emanating from Enceladus' south pole during the first three flybys of the Saturnian moon [Dougherty et $a l ., 2006]$. Stunning images of the plume, and the "tiger stripes" where the plume originates, were captured by the Imaging Science Subsystem (ISS) [Porco et al., 2006], and the Composite Infrared Spectrometer (CIRS) detected thermal emission from the "tiger stripes" [Spencer et al., 2006]. Of particular interest is the interpretation of data taken by the Ion and Neutral Mass Spectrometer (INMS) [Waite et al., 2006]. Waite et al. [2006] use a Monte Carlo simulation with a weak gravitational field and two particle sources, one uniform global source and one centered on the south pole. By tuning these sources, they get reasonable agreement with Cassini INMS data and net source between $1.0 \times$ $10^{26}$ to $3.0 \times 10^{27}$ molecules $\mathrm{s}^{-1}$. INMS data estimates that water accounts for 90.70 to 91.50 percent of the composition. Cassini's Ultraviolet Imaging Spectrograph (UVIS) observed stellar occultation of Enceladus, providing valuable information on the nature of the plume [Hansen et al., 2006]. Analyzing this data, Hansen et al. [2006] infer a column density of $1.5 \times 10^{16} \mathrm{~cm}^{-2}$. Assuming that the density corresponds to a vapor equilibrium, and the gas velocity is at least the thermal velocity, they arrive at a source rate of $5 \times 10^{27}$ molecules s ${ }^{-1}$. These recent measurements establish Enceladus as a significant mass source to Saturn's magnetosphere.

[6] The moon Titan is another mass source for Saturn's magnetosphere. Shematovich et al. [2003] and Michael et al. [2005] use one- and three-dimensional Monte Carlo simulations to quantify the source of neutral nitrogen resulting from photon and ion bombardment of the upper atmosphere. Smith et al. [2004] combine the results of these studies to estimate the net source rate of nitrogen atoms and molecules from Titan to be about $3.55 \times 10^{25} \mathrm{~s}^{-1}$, far less than the Enceladus source determined above. They use this as input to 3-D Monte Carlo simulations to determine the morphology of the Titan torus. Smith et al. [2004] also mention that the neutral density in the Titan torus is larger than the Voyager-based ion density.

[7] Recent observations of the plasma composition of Saturn's magnetosphere indicate that $\mathrm{N}^{+}$may not be as significant as previously thought. Young et al. [2005] present CAPS/IMS data from Cassini's initial orbit and note that $\mathrm{N}^{+}$was not detected in the region of Titan's orbit either on the inbound or outbound pass. They do note, however, that the spacecraft was significantly distant from Titan's orbital plane; so the lack of nitrogen ions may not be unreasonable. Smith et al. [2005] also study CAPS/IMS data and determine that that $\mathrm{N}^{+}$exists in Saturn's inner magnetosphere. The detected nitrogen ions are found to be close to local corotational energies and therefore are unlikely to be due to escape from Titan but are probably created locally. More work needs to be done to understand the source, and significance, of the nitrogen ions in Saturn's magnetosphere. However, current evidence seems to indi- cate that the Titan source is one to two orders of magnitude less than the icy satellite and Enceladus source.

[8] While the icy satellite and Enceladus sources of plasma are quite significant in the inner and outer magnetosphere, the ionosphere may play a role in the outer magnetosphere through the polar wind. The polar wind refers to the supersonic outflow of particles along open magnetic field lines at high latitude and can account for an important ionospheric source. First suggested by Axford [1968] and Banks and Holzer [1968] and demonstrated experimentally by Hoffman [1970], many models of the terrestrial polar wind have been developed over the years. Gombosi et al. [1985] introduce a time dependent field aligned model that has been subsequently used to investigate the importance of many physical parameters on the polar wind. An encompassing history of this model is presented in section 2 .

[9] Many other terrestrial field aligned models were developed, utilizing generalized transport, fluid, and kinetic approaches. For instance, Schunk [1981] uses a timeindependent formulation based on the 13 moment transport equations. By allowing 13 moments for electrons and fewer for ions, they could study electron anisotropies and simulate a supersonic polar wind. Mitchell and Palmadesso [1983] and Ganguli et al. [1987], introduce time-dependent models using 13 and 16 moment solutions, respectively. A collisional kinetic model of the polar wind is presented by Pierrard and Lemaire [1998] and used to study the escape of $\mathrm{H}^{+}$. A good review of polar wind models and measurements can be found in the work of Ganguli [1996].

[10] Terrestrial polar wind models demonstrate that several nonclassical effects can impact polar wind results significantly. Centrifugal acceleration resulting from flux tube convection across the polar cap can contribute to outflow of oxygen ions [Cladis, 1986; Horwitz et al., 1994]. Gombosi and Killeen [1987] demonstrate that friction heating can also lead to transient outflow of oxygen ions. As demonstrated by Ganguli and Palmadesso [1987] and others, wave particle interaction can also affect plasma outflow. Indeed, Barakat and Barghouthi [1994] use a Monte Carlo model to establish a strong connection between the flux oxygen ions and wave-particle interaction. In some cases the polar wind distribution can become nonMaxwellian. Barakat et al. [1995] show that in the transition region where the flow becomes supersonic, the distribution function becomes double humped. Many of these nonclassical effects that are present at Earth may also have a significant impact at Saturn.

[11] Three dimensional models of terrestrial polar wind have also been developed. Schunk and Sojka [1989] solve along various field lines convecting throughout the polar cap potential pattern. By tracking these field lines, they are able to obtain a three dimensional description of the polar wind. Gardner and Schunk [2005] solve a fully threedimensional model instead of tracking individual flux tubes. They also include the neutral polar wind, or the neutral particles flowing with the polar wind as a result of charge exchange, in their model. Both of these models solve the continuity and momentum equations, and use an adiabatic equation of state. Also, a simplification of the perpendicular momentum equation is employed by setting the perpendicular velocity equal to the $\mathbf{E} \times \mathbf{B}$ drift. 
Table 1. Chemistry in the Saturn Polar Wind Model

\begin{tabular}{|c|c|c|}
\hline Reaction & Reaction Rate & Reference \\
\hline $\begin{array}{l}\mathrm{H}_{2}+\mathrm{h} \nu \rightarrow \mathrm{H}^{+}+\mathrm{H}+\mathrm{e}^{-} \\
\rightarrow \mathrm{H}_{2}^{+}+\mathrm{e}^{-} \\
\mathrm{H}+\mathrm{h} \nu \rightarrow \mathrm{H}^{+} \\
\mathrm{H}_{2} \mathrm{O}+\mathrm{h} \nu \rightarrow \mathrm{H}^{+}+\mathrm{OH}+\mathrm{e}^{-} \\
\mathrm{H}^{+}+\mathrm{H}_{2}(\nu \geq 4) \rightarrow \mathrm{H}_{2}^{+}+\mathrm{H} \\
\mathrm{H}_{2}^{+}+\mathrm{H}_{2} \rightarrow \mathrm{H}_{3}^{+}+\mathrm{H} \\
\mathrm{H}^{+}+\mathrm{H}_{2}+\mathrm{M} \rightarrow \mathrm{H}_{3}^{+}+\mathrm{M} \\
\mathrm{H}^{+}+\mathrm{CH}_{4} \rightarrow \mathrm{CH}_{3}^{+}+\mathrm{H}_{2} \\
\rightarrow \mathrm{CH}_{4}^{+}+\mathrm{H} \\
\mathrm{H}_{3}^{+}+\mathrm{CH}_{4} \rightarrow \mathrm{CH}_{5}^{+}+\mathrm{H}_{2} \\
\mathrm{H}_{3}^{+}+\mathrm{H}_{2} \mathrm{O} \rightarrow \mathrm{H}_{3} \mathrm{O}^{+}+\mathrm{H}_{2} \\
\mathrm{H}^{+}+\mathrm{H}_{2} \mathrm{O} \rightarrow \mathrm{H}_{2} \mathrm{O}^{+}+\mathrm{H} \\
\mathrm{H}^{+}+\mathrm{e}^{-} \rightarrow \mathrm{H}+\mathrm{h} \nu \\
\mathrm{H}_{3}^{+}+\mathrm{e}^{-} \rightarrow \mathrm{H}_{2}+\mathrm{H} \\
\rightarrow \mathrm{H}+\mathrm{H}+\mathrm{H}\end{array}$ & $\begin{array}{c}1.9 \times 10^{-11} \mathrm{~s}^{-1} \\
9.9 \times 10^{-10} \mathrm{~s}^{-1} \\
1.0 \times 10^{-9} \mathrm{~s}^{-1} \\
4.2 \times 10^{-10} \mathrm{~s}^{-1} \\
\text { see text } \\
2.0 \times 10^{-9} \mathrm{~cm}^{3} \mathrm{~s}^{-1} \\
3.2 \times 10^{-29} \mathrm{~cm}^{6} \mathrm{~s}^{-1} \\
3.69 \times 10^{-9} \mathrm{~cm}^{3} \mathrm{~s}^{-1} \\
0.81 \times 10^{-9} \mathrm{~cm}^{3} \mathrm{~s}^{-1} \\
2.4 \times 10^{-9} \mathrm{~cm}^{3} \mathrm{~s}^{-1} \\
5.3 \times 10^{-9} \mathrm{~cm}^{3} \mathrm{~s}^{-1} \\
8.2 \times 10^{-9} \mathrm{~cm}^{3} \mathrm{~s}^{-1} \\
1.91 \times 10^{-10} T_{e}^{-0.7} \mathrm{~cm}^{3} \mathrm{~s}^{-1} \\
7.62 \times 10^{-7} T_{e}^{-0.5} \mathrm{~cm}^{3} \mathrm{~s}^{-1} \\
9.7 \times 10^{-7} T_{e}^{-0.5} \mathrm{~cm}^{3} \mathrm{~s}^{-1}\end{array}$ & $\begin{array}{c}\text { Moses and Bass, 2000] } \\
{[\text { Moses and Bass, 2000] }} \\
{[\text { Moses and Bass, 2000] }} \\
{[\text { Moses and Bass, 2000] }} \\
- \\
{[\text { Nagy, 1987], [Anichich, 1994] }} \\
{[\text { Capone et al. }, 1977]} \\
{[\text { Kim and Fox, 1994] }} \\
{[\text { Kim and Fox, 1994] }} \\
{[\text { Anichich, 1994] }} \\
{[\text { Anichich, 1994] }} \\
{[\text { Anichich, 1994] }} \\
{[\text { Kim and Fox, 1994] }} \\
{\left[\text { Kim and Fox, 1994] }{ }^{\mathrm{a}}\right.} \\
{[\text { Kim and Fox, 1994] }]^{\mathrm{a}}}\end{array}$ \\
\hline
\end{tabular}

[12] These models have been developed for the Earth and have yielded a wealth of information for understanding the polar wind. The chemistry and plasma constituents are different between the Earth and other magnetized planets, but the fundamental physical processes remain the same. Indeed, Nagy et al. [1986] show that the polar wind may be a significant source of plasma to the Jovian magnetosphere. Therefore the polar wind at Saturn may behave similarly to the terrestrial polar wind.

[13] Only one study of the polar wind at Saturn exists. Frey [1997] studies the polar wind at Saturn between 1400 and $8000 \mathrm{~km}$ above the 1 bar level. The study demonstrates the dependance of the polar wind on the neutral atmosphere and water and methane content. Notably, the larger neutral temperature raise the density of $\mathrm{CH}_{4}$ at the lower boundary and reduce the net plasma density. Furthermore, a flux of polar wind plasma between $10^{7}$ and $10^{8} \mathrm{~cm}^{-2} \mathrm{~s}^{-1}$ is estimated. Frey [1997] also considers the time variable nature of the polar wind. Simulating a flux tube that crosses from the dayside and convects to the nightside, they start from a steady state solution and turn off photoionization for a number of hours. Unfortunately, the altitude range is too small to observe a realistic sonic transition, making the plasma source difficult to characterize.

[14] Our study attempts to quantify the source of ionospheric plasma to the Saturnian magnetosphere. To do this, we introduce the Polar Wind Outflow Model (PWOM), a multifluid, first principles, model of Saturn's polar wind. The PWOM builds on previous work by extending the altitude range, the parameter range, improving the numerical scheme, and extending the physical validity of the model. Moreover, we consider an altitude range from 1400 to $61,000 \mathrm{~km}$ above the 1 bar level and a variety of possible neutral atmospheres. The neutral atmospheres, described in section 4, correspond to a selection of neutral temperatures ranging from $420 \mathrm{~K}$ to $1500 \mathrm{~K}$. Examining several possible parameters yields a range for the polar wind source at Saturn.

\section{Model Description}

[15] The Polar Wind Outflow Model (PWOM) solves the gyrotropic continuity, momentum, and energy equations that describe the supersonic ion outflow along open magnetic field lines in the polar region. The PWOM can simulate the polar winds of Earth and Saturn. At Earth the behavior of three ion species, $\mathrm{O}^{+}, \mathrm{H}^{+}$, and $\mathrm{He}^{+}$are considered, while at Saturn only two species, $\mathrm{H}^{+}$and $\mathrm{H}_{3}^{+}$, are considered. The model assumes a stationary neutral atmosphere. Ranging in altitude from $250 \mathrm{~km}$ to $8000 \mathrm{~km}$ for the Earth version, or 1400 to $61,000 \mathrm{~km}$ for the Saturn version, the PWOM has its lower boundary set in a reservoir at chemical and thermal equilibrium, while the top boundary is at considerably lower pressure, thus creating a transonic outflow to a low-pressure external medium. The considerable altitude range covers two different regimes; the collision and chemistry dominated low altitude, and the expansion dominated high altitude. Furthermore, the ambipolar electric field is calculated at every time step and is a major contributor to ion outflow. Other physical effects that are included in the PWOM, are topside electron heating, photoionization, and the expanding cross-sectional area of the magnetic flux tube. The ability to include field-aligned currents is present but not used in the current study. Energetic particle precipitation is included in the Earth version of the PWOM but has not yet been added to the Saturn version.

[16] First developed to describe the Earth's polar wind by Gombosi et al. [1985], the PWOM has undergone many improvements and has been used to investigate many features of the polar wind over the years. First, Gombosi [1988] added the ability to study field-aligned currents. Then, Cannata and Gombosi [1989] used this model to investigate the effect of solar cycle on the polar wind. These results were latter found to be qualitatively consistent with measurements taken by the Akebono satellite in a paper by Abe et al. [2004]. Gombosi et al. [1991] included helium ions into the model. More recently we have replaced the first-order Godunov solver with a more accurate secondorder Rusanov solver, use point-implicit discretization for the stiffest source terms, and expanded the model to work at Saturn.

[17] Modeling the polar wind at Saturn requires modifying the chemistry and collision routines as well as the neutral atmosphere. We use the chemistry model described in Table 1 . Note that $\mathrm{H}^{+}$is primarily due to photochemistry 
through dissociative ionization of $\mathrm{H}_{2}$, while $\mathrm{H}_{3}^{+}$is generated by the reaction between $\mathrm{H}_{2}$ and $\mathrm{H}_{2}^{+}$and the three body reaction involving $\mathrm{H}^{+}$and $\mathrm{H}_{2}$. Furthermore, the loss of $\mathrm{H}_{2}^{+}$ occurs so rapidly that we do not keep track of it in the model. For the current version of the code, the photoionization rates are kept constant with altitude. Solar zenith angle and altitude dependent photoionization rates will be included in future versions of the model. The chemical kinetic model is solved explicitly at all altitudes.

[18] We also include the loss of $\mathrm{H}^{+}$with vibrationally excited $\mathrm{H}_{2}$, as seen in Table 1. McElroy [1973] first suggested that this reaction is exothermic for vibrational levels greater than or equal to 4 . Work by Cravens [1987] and Majeed et al. [1991] demonstrate that vibrationally excited $\mathrm{H}_{2}$ may be a significant loss mechanism for $\mathrm{H}^{+}$in the Jovian and Saturnian ionospheres. However, the PWOM does not track the $\mathrm{H}_{2}(\nu \geq 4)$ density. Instead, we follow the example of Moses and Bass [2000] and use a effective reaction rate defined by

$$
k_{\text {effective }}=2 \times 10^{-9} \sum_{\nu=4}^{8} \exp \frac{-\left(E_{\nu} E_{0}\right)}{k T_{\nu}}
$$

Where the effective reaction rate uses the entire molecular hydrogen density, as opposed to the $\mathrm{H}_{2}(\nu \geq 4)$ population, and the vibrational temperatures in equation (1) are taken from Majeed et al. [1991].

[19] The five-moment gyrotropic transport equations used in the model are [Gombosi and Nagy, 1989]:

$$
\begin{gathered}
\frac{\partial}{\partial t}\left(A \rho_{i}\right)+\frac{\partial}{\partial r}\left(A \rho_{i} u_{i}\right)=A S_{i} \\
\frac{\partial}{\partial t}\left(A \rho_{i} u_{i}\right)+\frac{\partial}{\partial r}\left(A \rho_{i} u_{i}^{2}\right)+A \frac{\partial p_{i}}{\partial r}=A \rho_{i}\left(\frac{e}{m_{i}} E_{\|}-g\right)+A \frac{\delta M_{i}}{\delta t} \\
+A u_{i} S_{i}
\end{gathered}
$$

The subscript " $i$ " and the subscript "e" refer to the ion and electron species, respectively. With regard to the other symbols, $m$ is molecular mass, $\rho$ is mass density, $u$ is velocity, $T$ is temperature, $p$ is pressure, $e$ is particle charge, $r$ is the distance along the field line, $\mathrm{A}$ is the cross-sectional area, $\kappa$ is the heat conductivity, $\gamma$ is the specific heat ratio, $\mathrm{k}$ is Boltzmann's constant, $E_{\|}$is the ambipolar electric field, $g$ is the gravitational acceleration, $S$ is the mass production

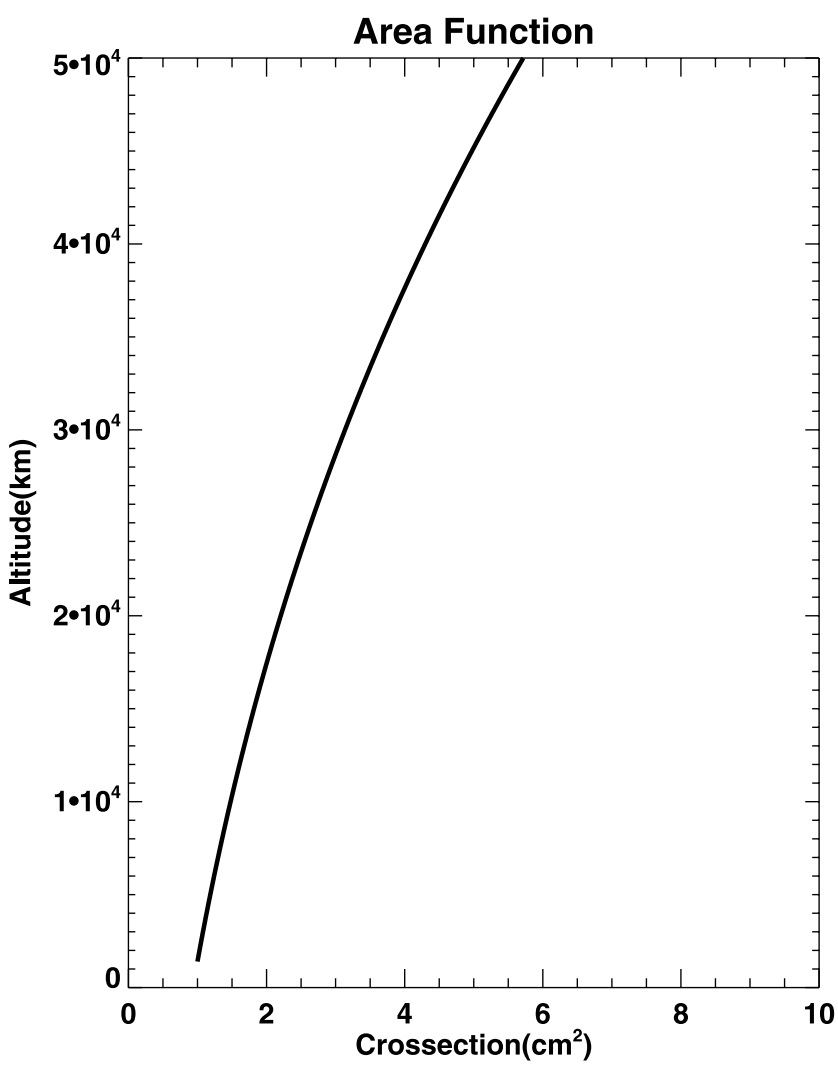

Figure 1. The cross-sectional area of a magnetic flux tube that has been normalized to have an area of $1 \mathrm{~cm}^{2}$ at the bottom of the simulation.

rate, $\frac{\delta M}{\delta t}$ is the momentum exchange rate, given by Schunk and Nagy [2000] as

$$
\frac{\delta M_{i}}{\delta t}=-\sum_{j} \rho_{i} \nu_{i j}\left(u_{i}-u_{j}\right)
$$

and $\frac{\delta E}{\delta t}$ is the energy exchange rate, given by Schunk and Nagy [2000] as

$$
\frac{\delta E_{i}}{\delta t}=\sum_{j} \frac{\rho_{i} \nu_{i j}}{m_{i}+m_{j}}\left[3 k\left(T_{j}-T_{i}\right)+m_{j}\left(u_{i}-u_{j}\right)^{2}\right]
$$

Here we assume that the neutrals are at rest. The gyrotropic transport equations (2) through (5) depend on the crosssectional area $A$. $A$ is inversely proportional to the magnetic field strength, and using a dipole assumption we have

$$
A=\alpha r^{3}
$$

where $\alpha$ is a constant. Figure 1 plots an example of the cross-sectional area of a flux tube.

[20] Equations (2) through (4) refer to the continuity, momentum, and energy equations respectively. Equation (5) determines the ambipolar electric field. Note, that the first term in (5), dependent on the electron pressure gradient, is the dominant term and frequently used by other models for the electric field. 
[21] Unlike the ions, the electrons are not solved for using the transport equations. Rather, they are solved using charge neutrality, a steady-state electron velocity assumption, and an energy equation:

$$
\begin{gathered}
n_{e}=\sum_{i} n_{i} \\
u_{e}=\frac{1}{n_{e}}\left(\sum_{i} n_{i} u_{i}-\frac{j}{e}\right) \\
j=j_{0} \frac{A_{0}}{A} \\
\rho_{e} \frac{\partial T_{e}}{\partial t}=\left(\gamma_{e}-1\right) \frac{m_{e}}{k A} \frac{\partial}{\partial r}\left(A \kappa_{e} \frac{\partial T_{e}}{\partial r}\right)-\rho_{e} u_{e} \frac{\partial T_{e}}{\partial r} \\
-T_{e}\left[S_{e}+\frac{\gamma_{e}-1}{A} \rho_{e} \frac{\partial}{\partial r}\left(A u_{e}\right)\right]+\left(\gamma_{e}-1\right) \frac{m_{e}}{k} \frac{\delta E_{e}}{\delta t}
\end{gathered}
$$

where $j$ is the current density, and the subscript 0 represents the value taken at a reference altitude. Expression (11) enforces conservation of field aligned currents. Note that the current density is set to zero for the purposes of this study, however future work may include the effect of field-aligned currents. Equation (9) represents the quasi-neutrality of the plasma and substitutes for the continuity equation. Similarly, equation (10) obtains the electron velocity from the ion flux and current and takes the place of the electron momentum equation. Finally, Equation (12) obtains the electron temperature from conduction, advection, adiabatic heating, and energy transfer due to chemical reactions.

\section{Numerical Schemes}

[22] This section describes the numerical schemes used in the PWOM to solve the equations presented in the previous section. The discretization is first-order in time and secondorder in space. We use operator splitting to solve the heat conduction separately from the rest of the equation. The heat conduction is discretized by the standard implicit Crank-Nicolson scheme.

[23] The advection terms are solved with the spatially second-order Total Variation Diminishing (TVD) LaxFriedrichs scheme with some minor modifications. The primitive variables $U$ (densities, velocities and pressures) are extrapolated to the cell faces from the adjacent cell centers.

[24] We apply limited reconstruction [VanLeer, 1979] to obtain the left and right face values

$$
\begin{gathered}
U_{j+1 / 2}^{L}=U_{j}+\frac{1}{2} \bar{\Delta} U_{j} \\
U_{j+1 / 2}^{R}=U_{j+1}-\frac{1}{2} \bar{\Delta} U_{j}
\end{gathered}
$$

where $j+1 / 2$ is the cell interface between the cells indexed by $j$ and $j+1$, and $\bar{\Delta} U_{j}$ is the limited slope of the variable. We use a modified monotized central limiter. If the left and right slopes $\Delta U_{j-1 / 2}=U_{j}-U_{j-1}$ and $\Delta U_{j+1 / 2}=U_{j+1}-U_{j}$ have opposite signs, the limited slope is zero. Otherwise we take

$$
\bar{\Delta} U_{j}=\operatorname{sgn} \Delta U_{j+1 / 2} \min \left(\beta\left|\Delta U_{j-1 / 2}\right|, \beta\left|\Delta U_{j+1 / 2}\right|, \frac{\left|U_{j+1}-U_{j-1}\right|}{2 \Delta x_{j}}\right)
$$

where sgn is the sign function and $1 \leq \beta \leq 2$ is an adjustable parameter. We found that $\beta=1.5$ produces robust and accurate results.

[25] The fluxes are calculated from the average of the left and right face values and a second-order dissipative LaxFriedrichs flux is added to obtain the numerical flux function

$$
F_{j+1 / 2}=\frac{F\left(U^{L}\right)+F\left(U^{R}\right)}{2}-\alpha\left(U_{j+1 / 2}^{R}-U_{j+1 / 2}^{L}\right)
$$

where $F$ is the physical flux function and we use

$$
\alpha=0.45 \frac{\Delta x}{\Delta t}
$$

The coefficient is reduced to 0.45 from the standard 0.5 because the cell area varies with altitude. Using the numerical flux function, the conservative update is

$$
U_{j}^{*}=U_{j}^{n}-\Delta t \frac{A_{j+1 / 2} F_{j+1 / 2}-A_{j-1 / 2} F_{j-1 / 2}}{V_{j}}+\Delta t S_{j}
$$

where $A_{j+1 / 2}$ is the cell face area, $V_{j}$ is the cell volume, $S_{j}$ contains all the explicitly treated source terms, and the superscript * refers to the fact that some of the source terms are yet to be added.

[26] The ion-neutral momentum transfer term in equation (6) and the corresponding ion-neutral energy transfer term in the equation (7) are evaluated implicitly so that a reasonable time step can be used. Since there are no spatial derivatives involved, the implicit equations can be solved easily. First, the momentum of species $i$ is updated as

$$
(\rho u)_{i}^{n+1}=\frac{(\rho u)_{i}^{*}}{1+\Delta t \sum_{j} \nu_{i j}}
$$

where $(\rho u)^{*}$ already contains the contributions from advection and other source terms, and $\nu_{i j}$ is the collisional frequency between ion species $i$ and neutral species $j$. Here we exploit the fact that the neutral velocity is zero, but the formula can be easily generalized for nonzero $v_{j}$. Using the fully updated velocity and the partially updated energy, the partially updated pressure is

$$
p_{i}^{*}=\left(\gamma_{i}-1\right)\left[e_{i}^{*}-\frac{1}{2} \rho_{i}^{n+1}\left(u_{i}^{n+1}\right)^{2}\right]
$$

where $e$ is the energy density obtained from the conservative update. Then the pressure is updated implicitly as

$$
p_{i}^{n+1}=\frac{p_{i}^{*}+\left(k / m_{i}\right) \rho_{i}^{n} \Delta t \sum_{j} \mu_{i j} \nu_{i j} T_{j}^{n}}{1+\Delta t \sum_{j} \mu_{i j} \nu_{i j}}
$$


where $\mu_{i j}=2\left(m_{i}\right) /\left(m_{i}+m_{j}\right)$ is the effective mass ratio, and the sum is over neutral species indexed by $j$.

[27] The numerical scheme described above is implemented on a grid with an expanding cross-sectional area described by equation (8). The bottom of the grid is located at $1400 \mathrm{~km}$ above the $1 \mathrm{bar}$ level, and the top of the grid is located at about 1 Saturn Radius above the 1 bar level. Eight hundred cells are used with a grid spacing of $75 \mathrm{~km}$. This resolution is shown by a grid convergence study to be sufficient. Grids with 200, 400, 800, and 1500 cells are considered, and little difference is found between a grid using 1500 cells and one using 800 cells. Therefore by using the 800 cell grid we can complete the simulations in less time with confidence that the solution is accurate. For the 800 cell grid we can use a time step of $0.1 \mathrm{~s}$.

\section{Neutral Background for Saturn}

[28] The PWOM requires the background neutral atmosphere as an input. For the Earth, the model uses the neutral background from MSIS 90. Unfortunately, a similar empirical model does not yet exist for Saturn. We therefore rely on analysis of the stellar occultation measurements of the Voyager 2 Saturn flyby, presented by Smith et al. [1983], and other estimates to create our neutral background.

[29] Most of the neutral constituents, as used in the PWOM, have their values defined at an altitude of $1400 \mathrm{~km}$, and are then extended to higher altitudes using a hydrostatic distribution. Close examination of Smith et al. [1983] demonstrates that the species independent hydrostatic assumption is reasonable above $1400 \mathrm{~km}$. The lower boundary densities of $\mathrm{H}_{2}$ and $\mathrm{H}$ are set, according to analysis of the afore mentioned stellar occultation of Smith et al. [1983], to $6.3 \times 10^{9} \mathrm{~cm}^{-3}$ and $5.0 \times 10^{7} \mathrm{~cm}^{-3}$ respectively. The density of $\mathrm{H}_{2} \mathrm{O}$ is set to $6.3 \times 10^{2} \mathrm{~cm}^{-3}$, which represents a flux of $10^{6}$ molecules $\mathrm{cm}^{-2} \mathrm{~s}^{-1}$ according to Majeed and McConnell [1991]. This influx of water is comparable to Infrared Space Observatory (ISO) measurements presented by Feuchtgruber et al. [1997]. Like $\mathrm{H}_{2}$ and $\mathrm{H}$, the density of $\mathrm{CH}_{4}$ is also taken from Smith et al. [1983]; but it is set to $1.6 \times 10^{8} \mathrm{~cm}^{-3}$ at $1000 \mathrm{~km}$ because that is the highest altitude for which measurements of $\mathrm{CH}_{4}$ are available.

[30] There are challenges in approximating the neutral atmosphere as described. The Voyager 2 stellar occultation was taken at low latitude, but the polar wind is a process that takes place in the open field line region at high latitude. Joule heating in particular can cause a strong increase in temperature in the polar region. Furthermore, the value of the exospheric neutral temperature, which affects the neutral scale height, is not well constrained. According to Smith et al. [1983], the neutral temperature is $420 \mathrm{~K}$; Festou and Atreya [1982] estimate the neutral temperature to be $800 \mathrm{~K}$. Preliminary work by Shemansky et al. [2005], using Cassini data, estimates the neutral temperature at $345 \mathrm{~K}$. The neutral temperature can also be estimated from the plasma temperature. At low altitudes, the neutral temperature and the plasma temperature should coincide. Nagy et al. [2005] use radio occultation measurements from Cassini to estimate a plasma temperature consistent with $1000 \mathrm{~K}$. Finally, Moore and Mendillo [2005] cite numerous studies to infer the range of topside plasma temperatures between approximately 260 and $1700 \mathrm{~K}$.

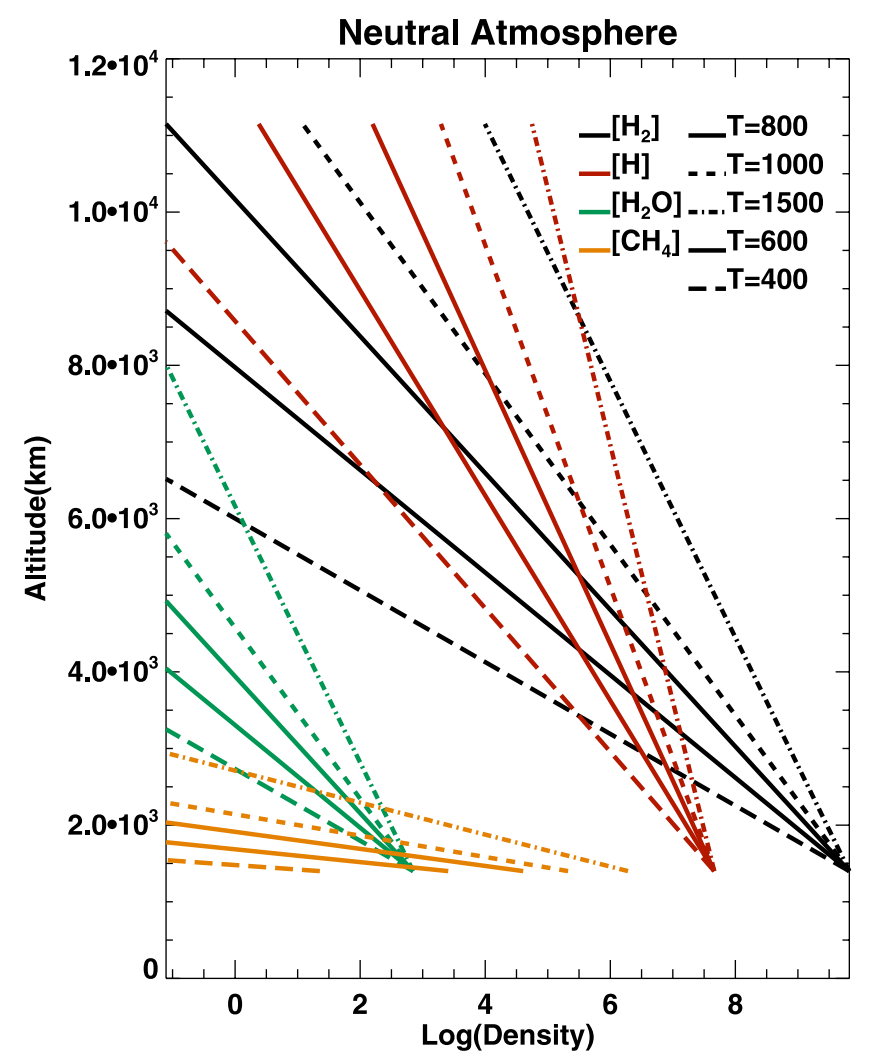

Figure 2. Plots of the various background neutral atmospheres considered.

[31] We consider a wide array of cases in an attempt to span the parameter space of possible neutral temperatures. Figure 2 illustrates the various cases considered in this study. The neutral temperatures range from 420 to $1500 \mathrm{~K}$, effectively covering the range of possible neutral temperatures. In the future, a more sophisticated neutral atmosphere may be considered, but for the purposes of this study, a simple isothermal and hydrostatic model is sufficient.

\section{Results}

[32] Using the neutral atmosphere described in section 4, we run the PWOM until a steady state is achieved for a number of scenarios. In order to disregard the effect of the upper boundary, the results are only presented below $50,000 \mathrm{~km}$. Below $50,000 \mathrm{~km}$, the result is reasonably insensitive to the position of the boundary. We verify this assertion by extending the simulation domain up to about $71,000 \mathrm{~km}$, and comparing the results with the original simulation. We find that below an altitude of about 50,000 $\mathrm{km}$ the differences in the result are small. Density, electric field, and flux are presented for various neutral atmospheres corresponding to neutral temperatures 420, 600, 800,1000 , and $1500 \mathrm{~K}$.

[33] Figure 3 shows the ion densities for the various neutral temperatures. Changing the neutral temperature affects the neutral background, which in turn affects the ion density. Note that for lower neutral temperatures $\mathrm{H}^{+}$is the dominant ion, while for higher neutral temperatures $\mathrm{H}_{3}^{+}$ is the dominant ion. Which ion species dominates is very 

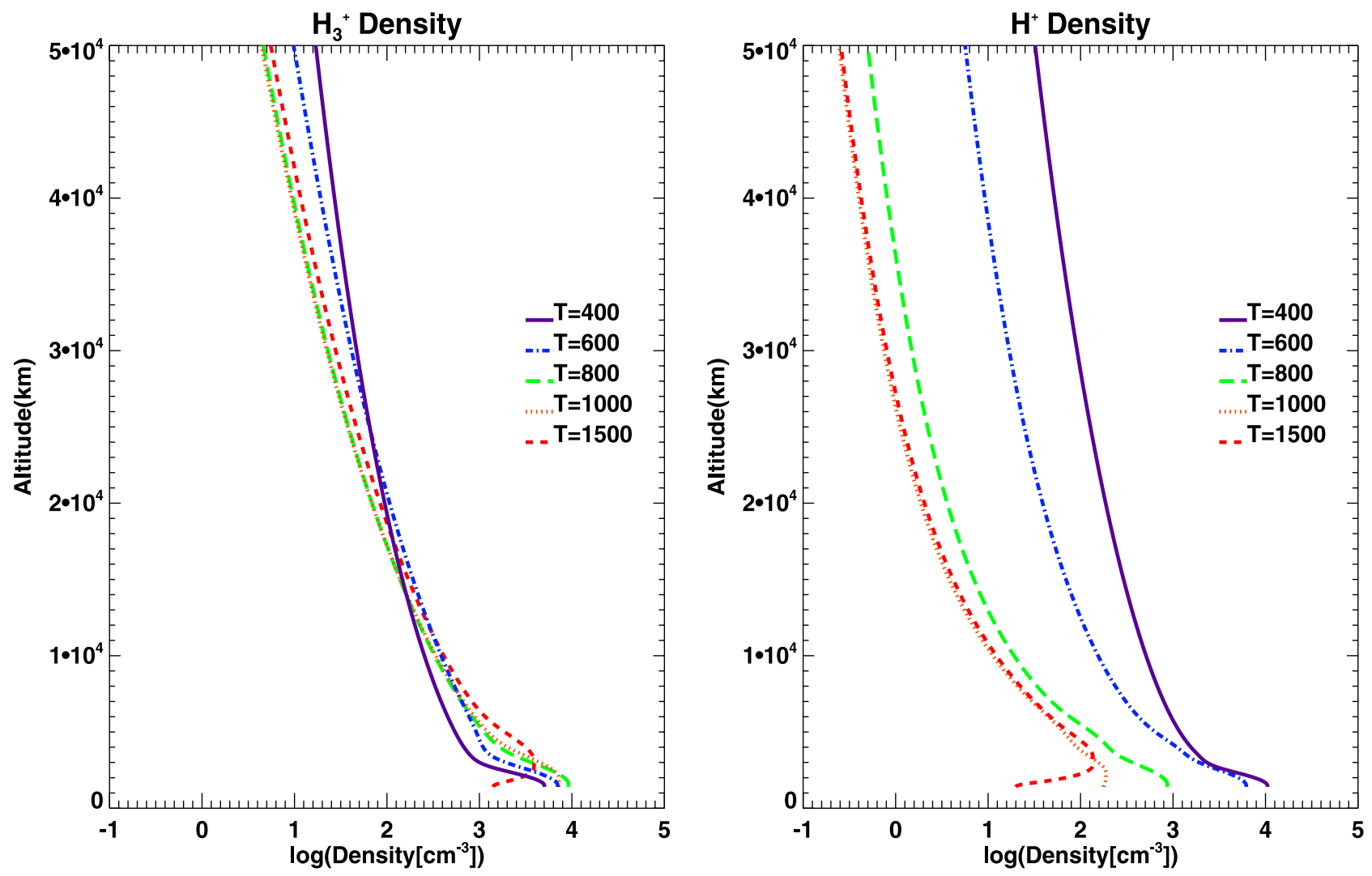

Figure 3. Plots of ion densities with $\mathrm{H}_{3}^{+}$on the left and $\mathrm{H}^{+}$on the right. Note that the neutral temperature affects the relative ion density.

important for the polar wind process. To a first approximation, the ambipolar electric field applies an upward force equal to about half the weight of the major ion. This is seen by keeping only the electron pressure gradient term in equation (5) and using a simple hydrostatic assumption. Therefore increasing the neutral temperature leads not only to an increase in the mass of the major plasma constituent but also leads to an increase in the ambipolar electric field. Figure 4 clearly demonstrates the calculated electric field's dependance on neutral temperature. While the peak electric field is larger for lower neutral temperatures, the electric field throughout most of the simulated range is smaller.

[34] The electron density calculated by the model is of interest for several reasons. Because of the quasi-neutrality condition imposed on the electrons, the electron density is representative of the total plasma density. It is also one of the few quantities for which high-latitude data is available. Indeed, the Voyager 1 ingress radio occultation profile, presented by Atreya et al. [1984], provides data at $71^{\circ} \mathrm{S}$ latitude. Unfortunately, several challenges exist to doing a direct data-model comparison. First, without exact knowledge of the neutral atmosphere, it is difficult to precisely reproduce the data. Furthermore, it is not clear that $71^{\circ} \mathrm{S}$ is at sufficiently high latitude to place the measurement in the polar cap. Despite these difficulties, we compare our modeled cases to the available data. Figure 5 directly compares the measured and calculated electron densities below $10,000 \mathrm{~km}$. The comparison shows agreement in overall features. Notably, the clear density peak followed by a sharp decrease is evident in both the data and the modeled cases. The change in slope of the density decrease is also evident in both the data and modeled cases; it is representative of the importance of the vertical transport of plasma. Many key differences are also seen in the comparison. For instance, some cases match the magnitude of the density peak, while others match the location, but none of the afore mentioned cases do both.

[35] The location of the density peak represents the altitude at which vertical transport and chemistry balance. Increasing the chemical source lowers altitude of the peak, while increasing vertical advection raises the altitude. These parameters are not independent; the low-temperature cases have increased vertical transport and increased source terms (due to decreased loss from $\mathrm{CH}_{4}$ ), while the higher temperature cases have decreased vertical transport and decreased source terms (due to increased loss from $\mathrm{CH}_{4}$ ). It is expected that the peak density will always occur above the lower boundary of the model. In the high temperature cases this occurs, but for temperatures below $800 \mathrm{~K}$ we find that the density peaks at the boundary. There are several reasons that this could occur. One reason, is that the neutral densities at these latitudes are not well constrained, and changes will have a significant effect on the chemical source. Another reason has to do with a limitation of the model; the photoionization rate is not altitude dependent. As a result, the source at the lower boundary might be exaggerated, thereby lowering the peak. Future versions of the PWOM will include an altitude dependent photoionization 


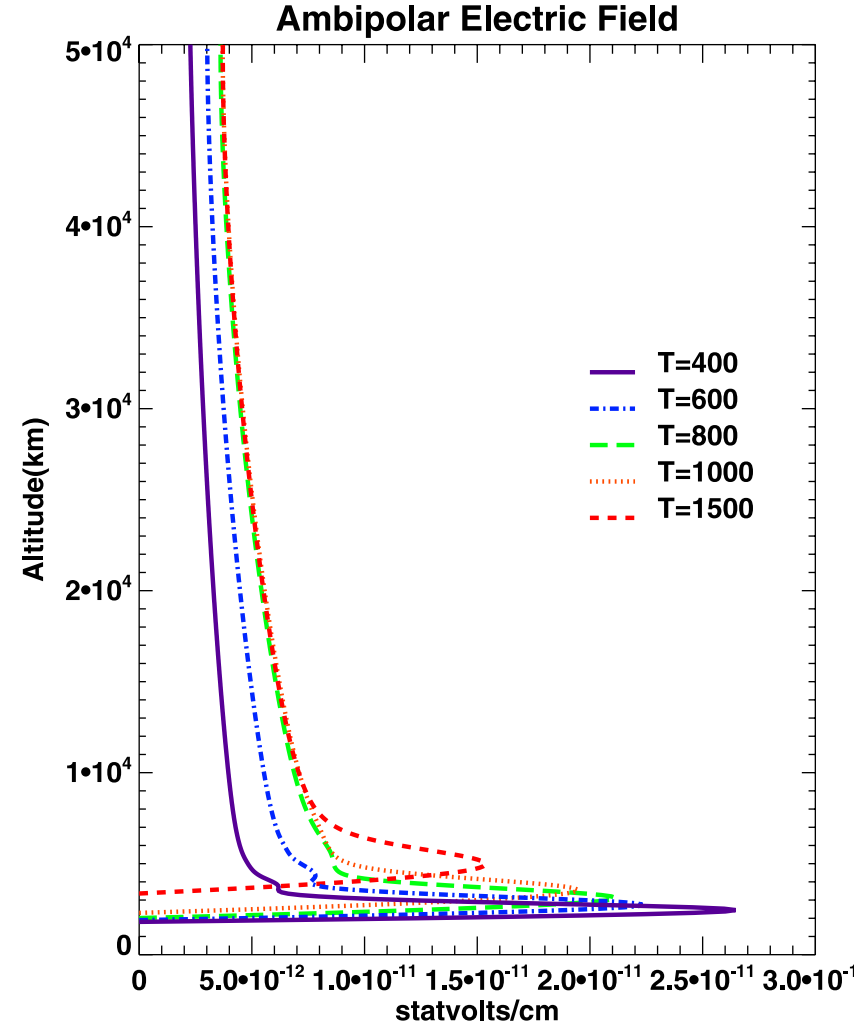

Figure 4. The plot demonstrates the ambipolar electric fields dependance on the neutral temperature. The cases with lower neutral temperatures have smaller electric field strength in the expansion region.

rate, but for obtaining a first estimate of the ionospheric source, the current model is adequate.

[36] Because none of the previous cases showed strong agreement with the only available data set, we have tuned the neutral atmosphere to obtain improved agreement. Labeled case 1000(b) in Figure 5, we consider an increase of a factor of 10 in $\mathrm{H}_{2}$ and 1000 in $\mathrm{H}_{2} \mathrm{O}$ density with a neutral temperature of $1000 \mathrm{~K}$, and find a better match with the observed electron density. Additionally, we let $\mathrm{H}_{2} \mathrm{O}$ decrease with a shorter scale height, reflecting that the neutral constituents are not well mixed at the altitudes considered. Increasing the $\mathrm{H}_{2}$ density could be reflective of the importance of the energy input from Joule heating, which Cowley et al. [2004b] estimate to be more than 10 times the average solar input. Also, the density of water is not based on in situ measurement but rather on remote measurements. The uncertainty of the estimate is therefore greater. Moreover, Connerney and Waite [1984] note that the influx of $\mathrm{H}_{2} \mathrm{O}$ is not spatially uniform. In particular, they note that at latitudes $\left(-38^{\circ},+44^{\circ}\right)$, which are magnetically connected to the inner $\mathrm{B}$ ring, the water influx may be approximately 50 times greater than the global average. The Voyager 1 data shown in Figure 5 was taken at $71^{\circ} \mathrm{S}$ which corresponds to $\mathrm{L} \approx 8.5$, or just past the edge of the $\mathrm{E}$ ring. Increasing the water density to this level has been studied by Majeed and McConnell [1991] and Moses and Bass [2000] and corresponds to an influx of about $10^{8}$ molecules $\mathrm{cm}^{-3}$ $\mathrm{s}^{-1}$. The flux is the largest considered in these studies but not outside the realm of possibilities. This demonstrates the importance of accurate knowledge of the neutral densities. Future high-latitude measurements by the Cassini spacecraft will assist in this endeavor.

[37] The ion temperatures, given in Figure 6, illustrate the interaction between the two different physical regimes of the polar wind: one dominated by collisions with the background neutral atmosphere and one that is expansion dominated. The relative size of the two regions is determined mainly by the neutral temperature. Larger neutral temperatures represent an increased neutral scale height, which gives rise to an expanded atmosphere. The result is to increase the region over which collisions are important. Hence the region over which the ion temperature corresponds to the neutral temperature also increases. This is seen in Figure 6; where ion temperatures match the neutral temperatures is the collisional region, and where the ion and neutral temperatures begin to depart is the expansion dominated region. Furthermore, the collisions at low altitude lead to frictional heating that increases the ion temperature. Adiabatic cooling at high altitude, due to expansion, counteracts the low-altitude frictional heating. A consequence of the low-altitude heating and high-altitude cooling is the formation of a temperature peak in Figure 6.

[38] Another interesting feature of the ion temperature is the nonlinear dependence on the neutral temperature. The lowest neutral temperature case in Figure 6 corresponds to the lowest peak ion temperature, but the highest neutral temperature case does not correspond to the highest peak

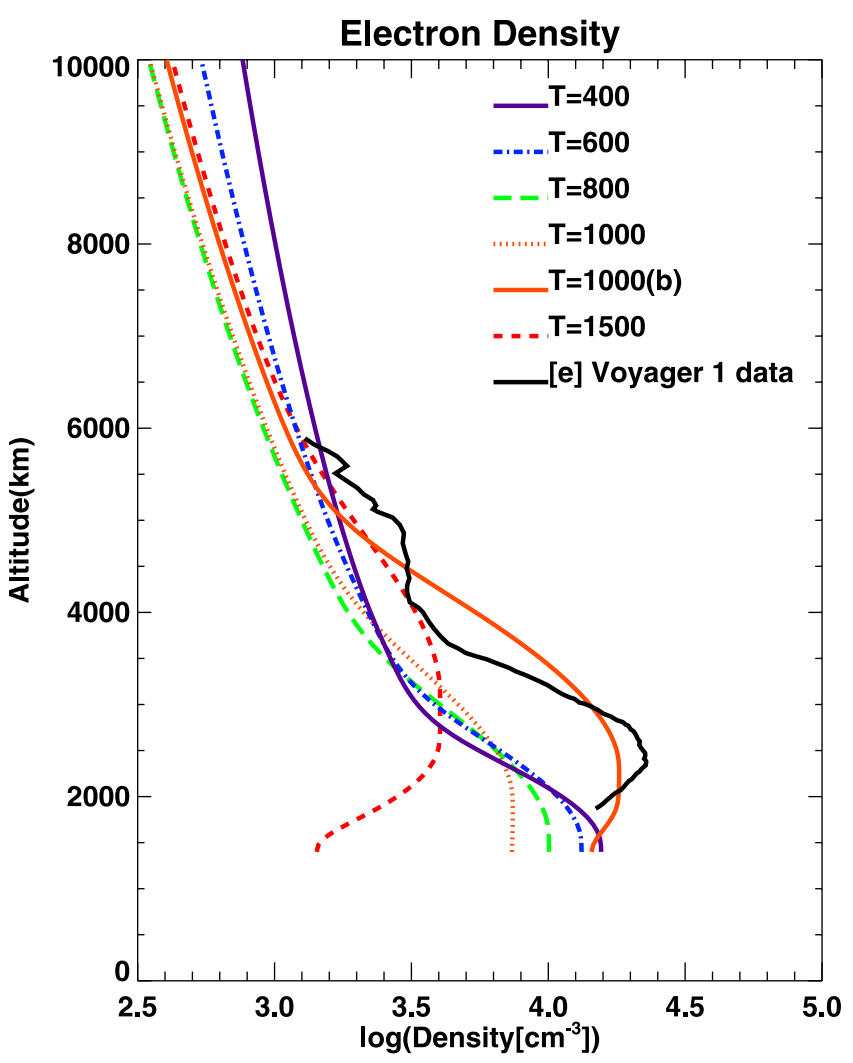

Figure 5. The plot demonstrates the electron densities dependence on the neutral temperature, and compares the result with Voyager 1 data taken at $71^{\circ} \mathrm{S}$. 

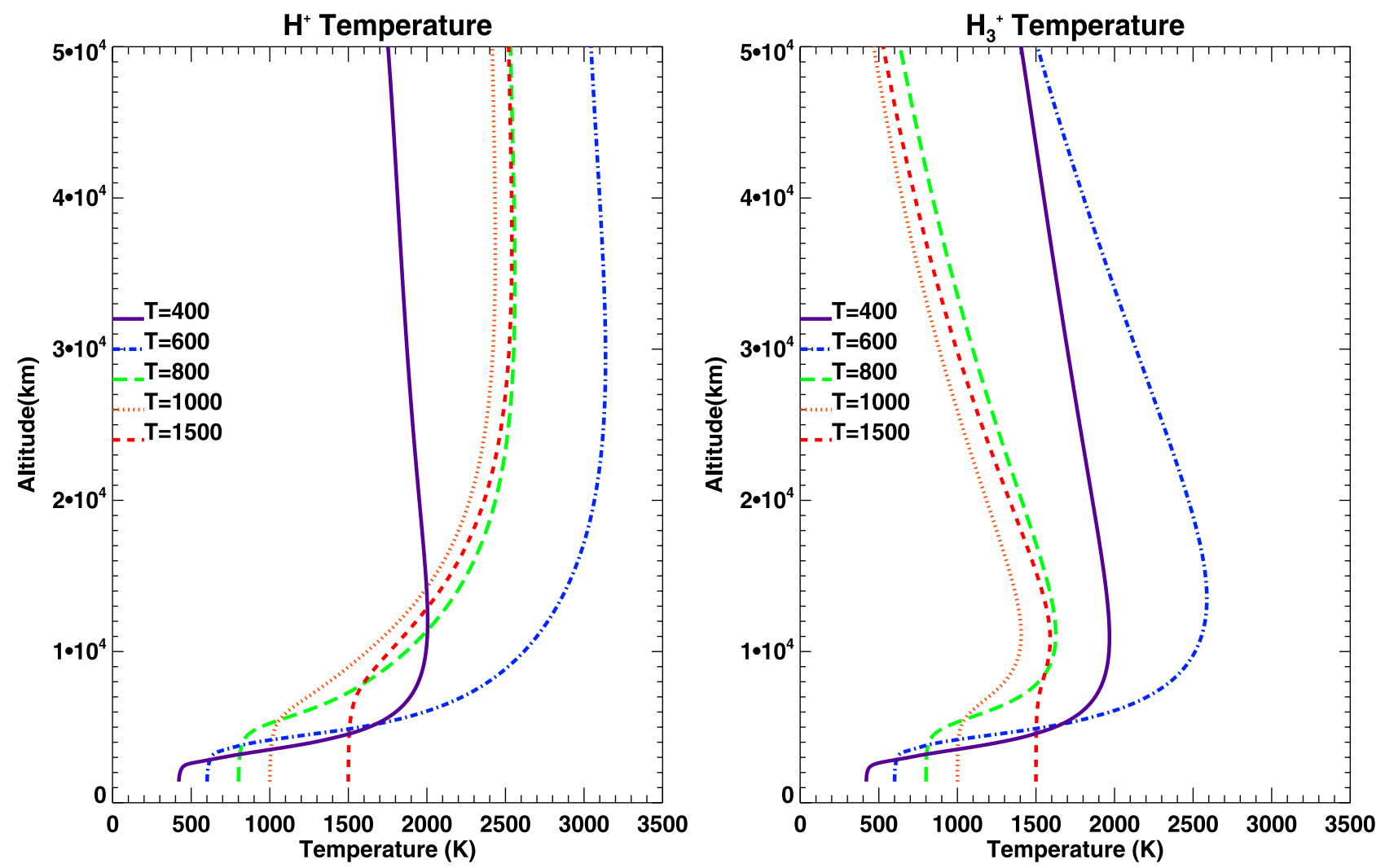

Figure 6. The plot on the left shows the ion temperature of $\mathrm{H}^{+}$. The plot on the right shows the plasma temperature of $\mathrm{H}_{3}^{+}$.

ion temperature. The heating depends on the two terms shown in equation (7). The first term is a frictional heating term, and the second term tries to keep the ion temperature coincident with the neutral temperature. However, only the first term raises the peak, and it depends on the collision frequency and velocity difference. The coldest neutral temperature corresponds to the case with the lowest collision frequency, but the largest velocity difference. The hottest neutral temperature corresponds to the case with the largest collision frequency, but the smallest velocity difference. By contrast, the middle temperature cases have reasonably large collision frequencies and velocity differences. The result is that the largest temperature peaks occur for temperatures in the middle of the range considered.

[39] Particle flux is the key quantity to consider when gauging the polar wind's importance as a mass source. In particular, equation (10) demonstrates that electron flux is equivalent to the net particle flux since field-aligned currents are not considered in this case. Figure 7 presents the electron flux multiplied by the cross-sectional area of a flux tube. Since we are using a gyrotropic assumption, particles may only be lost through chemical reactions. Therefore the number flux in a magnetic flux tube remains constant in the expansion dominated regime but is not constant at low altitudes. Figure 7 illustrates that above about $5000 \mathrm{~km}$ the number of particles flowing through the flux tube remains constant.

[40] As demonstrated above, the neutral temperature greatly influences the polar wind. Figure 7 illustrates the importance of the neutral temperature to the outflow of

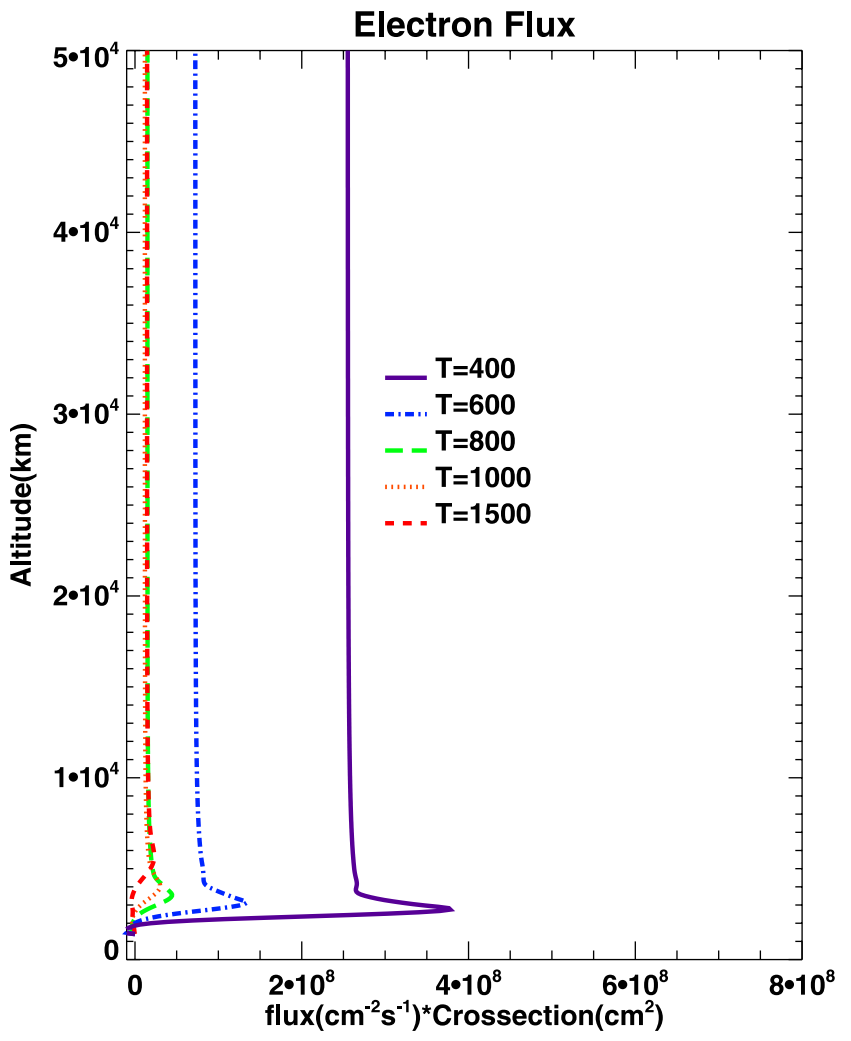

Figure 7. A plot of electron flux times the altitude dependent cross-sectional area normalized to $1 \mathrm{~cm}^{2}$ at $1400 \mathrm{~km}$. 
plasma. Notably, lower neutral temperatures translate to greater outflow than higher neutral temperatures. There are two main reasons for this behavior. First, because the low-temperature atmosphere is more compressed than a warmer atmosphere, the collisional region is smaller, reducing collisions that impede the flow at low altitudes. Second, a cooler neutral background allows less $\mathrm{CH}_{4}$ at the bottom of the model; as a consequence, the chemical loss of ions is reduced, leading to increased plasma densities. Therefore improved knowledge of the $\mathrm{CH}_{4}$ density is needed to fully understand the plasma densities and outflows.

[41] To translate flux into a net source rate for the magnetosphere, we must know the size of the polar cap. We obtain the polar cap area by using a Space Weather Modeling Framework [Tóth et al., 2005] simulation of Saturn's magnetosphere. In particular, we use simulations conducted by Hansen et al. [2005], and extract the total polar cap area for each of the seven bow shock crossing during the inbound leg of Cassini's Saturn orbit insertion, at times corresponding to 27/06/04: 0945, 1030, 1800, 2000, and 28/06/04: 0015, 0300, 0545. This process yields a range of values from $2.9 \times 10^{19} \mathrm{~cm}^{2}$ to $4.4 \times 10^{19} \mathrm{~cm}^{2}$ at $10,000 \mathrm{~km}$ above the one bar level. Multiplying the polar cap area from the MHD simulation by flux of polar wind plasma yields the particle source rate. From the PWOM, we estimate that the polar wind number flux is between $7.3 \times$ $10^{6}$ and $1.7 \times 10^{8} \mathrm{~cm}^{-2} \mathrm{~s}^{-1}$, yielding a particle source rate between $2.1 \times 10^{26}$ and $7.5 \times 10^{27} \mathrm{~s}^{-1}$.

\section{Discussion of Results and Future Work}

[42] The results presented in section 5 show that the magnitude of the polar wind source is between that of the icy satellites, calculated by Jurac and Richardson [2005], and of the Titan Torus, calculated by Smith et al. [2004]. However, the polar wind source must be considered in context. Figure 8 shows a schematic of the spatial distribution of various mass sources. Note that the polar wind is mostly important in the open field line region, whereas the source from the icy satellites is more important for the inner magnetosphere.

[43] Although the polar wind flows along open field lines, the plasma from the polar wind can make its way into lower latitudes. Consider, for example, an open field line about to reconnect in the tail. Such a field line has polar wind plasma flowing along it. After reconnection that same field line still has polar wind plasma flowing along it. Following the Dungey cycle [Dungey, 1961], that field line is now closed and begins to convect toward the dayside. Thus polar wind plasma can enter the closed field line region. We can estimate the efficiency of this process using a simple back-of-the-envelope calculation (S. Cowley, private communication, 2006). Jackman et al. [2004] find that the transpolar transit time can range from 1 to 8 days depending on solar wind conditions. Using an average polar wind velocity of $10 \mathrm{~km} \mathrm{~s}^{-1}$, we find that the polar wind plasma can flow 14 to 115 Saturn Radii along the field line before reconnection can occur. The length of Saturn's tail is not well determined, but global MHD simulations of Saturn's magnetosphere [Hansen et al., 2005, Figure 3] place the tail length at more than 65 Saturn Radii. Polar wind plasma that flows less than this distance before reconnection

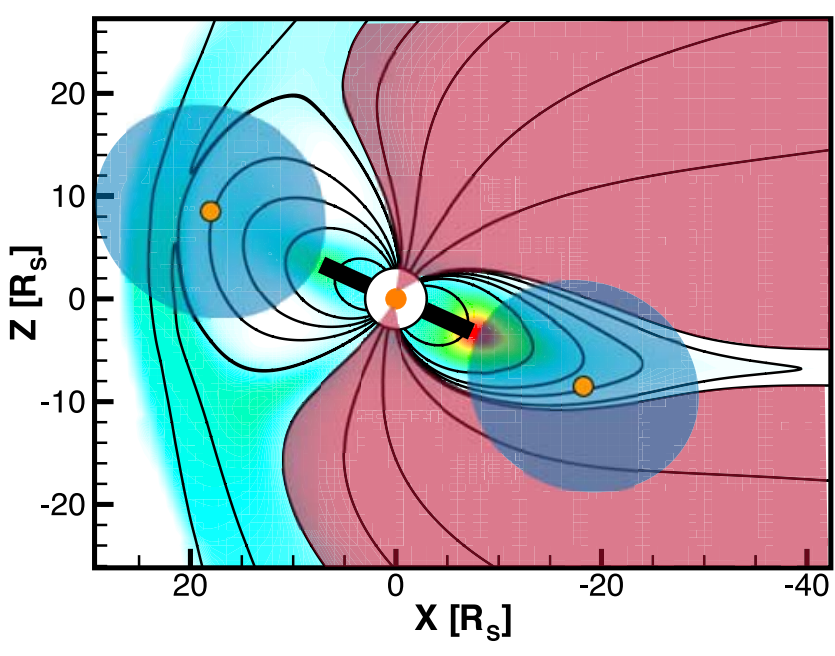

Figure 8. The schematic shows the location of three important mass sources to Saturn's magnetosphere. The E-ring and Enceladus are shown in black, and the Titan torus is shown as a blue oval around an orange dot at Titan's orbit. The region where the polar wind is important is shown in purple and represents the open field line regions.

occurs is trapped in the magnetosphere. Therefore at least 57 percent of all escaping polar wind plasma remains in the magnetosphere.

[44] It is clear from the simulations that the neutral background density and temperature strongly influence the polar wind outflow. Increased temperature can cause the atmosphere to heat up and heave up; the breadth of the collisional region increases, thereby constricting the outflow of plasma. Furthermore, changes in the neutral density at the bottom of the model affects the relative ion densities. Changing the relative ion densities changes the ambipolar electric field, which in turn modifies the outflow through the momentum equation. However, since the low temperature cases have a reduced electric field but also an increased net ion outflow, the ambipolar electric field in the expansion region is not the main controller of the outflow rate. Furthermore, the decreased density of $\mathrm{CH}_{4}$ in the low temperature cases are extremely important; reduced $\mathrm{CH}_{4}$ is a major contributor to increased ion densities, and as a result, increased flux.

[45] The fluxes calculated by the PWOM compare well with those calculated by Frey [1997], despite some significant differences. As mentioned earlier, Frey [1997] calculate a flux between $10^{7}$ and $10^{8} \mathrm{~cm}^{-2} \mathrm{~s}^{-1}$ which is comparable to what the PWOM predicts. The differences between the models arise from an improved chemical scheme, neutral atmopshere, and altitude range. The PWOM uses a photo-ionization rate of $1.9 \times 10^{-9}$ for the reaction $\mathrm{H}_{2}+\mathrm{h} \nu \rightarrow \mathrm{H}^{+}+\mathrm{H}+\mathrm{e}$, which is 5 times less than the value used by Frey [1997]. Since this reaction is a major source of $\mathrm{H}^{+}$, the reduced rate will lead to smaller densities. Moreover, the Frey [1997] model does not include loss of $\mathrm{H}^{+}$ with vibrationally excited molecular hydrogen. This is a major loss process and also serves to reduce the density of $\mathrm{H}^{+}$. Counteracting these effects is the significant reduction in the amount of water in the background neutral atmosphere. Our model uses a water flux that is comparable to 
Infrared Space Observatory (ISO) measurements as reported by Feuchtgruber et al. [1997]. In contrast, Frey [1997] use a much larger number inferred by Majeed and McConnell [1991]. Since water is a major loss of $\mathrm{H}^{+}$, reducing the amount of water bolsters the density of $\mathrm{H}^{+}$. Because some of our differences reduce the $\mathrm{H}^{+}$density, and some raise the density, our results are in reasonable agreement with those of Frey [1997].

[46] Direct comparison of our results with other Saturnian ionospheric models is difficult for several reasons. First, most models are low latitude models, and therefore do not include polar wind processes. Additionally, the altitude range considered by other models is very different, and diffusion is their main plasma transport process. Finally, low latitude models do not consider the range of neutral temperatures that may be available at high latitudes. Despite these differences, we can still make a qualitative comparison using the lowest temperature case considered by the PWOM at low-altitude (near $1400 \mathrm{~km}$ ). Relative ion densities at low altitudes in the lowest temperature case are in good agreement with the studies of Moses and Bass [2000] and Moore et al. [2004]. This region is well within the chemical equilibrium regime, and due to similar chemical models, good agreement is expected.

[47] A lack of measurements of the background neutral atmosphere at high latitude restricts the ability of the PWOM to go beyond understanding fundamental processes and making zeroth-order source estimates. The high-latitude atmosphere may vary significantly from that at low latitude. Increased solar zenith angle, and Joule heating are two physical effects that may cause such differences. As shown above, the polar wind at Saturn is heavily reliant on the neutral background; the uncertainties in the neutral background resulting from the lack of measurements can strongly influence the results. Future measurements by the Cassini spacecraft should help address the issues resulting from the sparsity of data.

[48] As alluded to in the introduction, several nonclassical effects may impact Saturn's polar wind. Centrifugal acceleration, frictional heating, and wave-particle interactions may enhance the flow and create transient features in the polar wind. These effects are outside the range of the PWOM, but we discuss their potential significance. We carry out a simple calculation to estimate the importance of centrifugal acceleration and frictional heating. Cowley et al. [2004a] estimate that the transpolar flow speed in the polar cap is approximately $200 \mathrm{~m} \mathrm{~s}^{-1}$. Therefore at the lower boundary of our model the centrifugal force is found to be $10^{5}$ times less than the gravitational force and is thus a small correction. By using equation (7), assuming that the neutrals are stationary and have the same temperature as the ions and that the number density is on the order of $10^{3} \mathrm{~cm}^{-3}$, we estimate that the energy input due to frictional heating is, at most, on the order of $10^{-13}$ Joules $\mathrm{m}^{-3}$. This could be a very significant effect leading to transient outflows. The region were frictional heating is important is spatially and temporally constrained and will require tracking the flux tube motion. Future versions of the PWOM should include this potentially important effect. The importance of waveparticle interactions to Saturn's polar wind is much harder estimate, and therefore more study is needed before any meaningful statement can be made.
[49] Further improvements to the model will allow us to expand upon the present study. In particular, including a solar zenith angle dependance on photoionization in the Saturn version of the PWOM will allow us to study the polar wind at various locations in the polar cap, and will allow improved density profiles. The inclusion of impact ionization will also be an improvement and will raise the source estimates calculated here. A more sophisticated chemical model should also be used. These improvements will increase the accuracy and scope of the model. We also plan to integrate the PWOM into the Space Weather Modeling Framework so that it can be used for the Earth simulations as well.

[50] Acknowledgments. This work has been supported at the University of Michigan by the NASA Cassini Project under contract JPL-1279089, NASA AISRP grant NNG04GP89G, and by DoD MURI grant F49620-01-1-0359. G. T. was partially supported by the Hungarian Science Foundation (OTKA, grant T047042). The authors would also like to thank S. Cowley for his helpful comments regarding trapping polar wind plasma and D. E. Shemansky for his useful insights regarding the chemical scheme.

[51] Wolfgang Baumjohann thanks Abdallah R. Barakat and another reviewer for their assistance in evaluating this paper.

\section{References}

Abe, T., A. W. Yau, S. Watanabe, M. Yamada, and E. Sagawa (2004), Long-term variation of the polar wind velocity and its implication for the ion acceleration process: Akebono/suprathermal ion mass spectrometer observations, J. Geophys. Res., 109, A09305, doi:10.1029/ 2003JA010223.

Anichich, V. G. (1994), Evaluated bimolecular ion-molecule gas phase kinetics of positive ions for use in modeling the chemistry of planetary atmospheres, cometary comae, and interstellar clouds, J. Phys. Chem. Ref. Data, 22, 1469.

Atreya, S. K., T. M. Donahue, A. F. Nagy, J. H. Waite, and J. C. McConnell (1984), Saturn, in Theory, Measurements, and Models of the Upper Atmosphere and Ionosphere of Saturn, edited by T. Gehrels and M. S. Matthews, pp. 239-277, Univ. of Ariz. Press, Tucson, Ariz.

Axford, W. I. (1968), The polar wind and the terrestrial helium budget, J. Geophys. Res., 73, 6855.

Banks, P. M., and T. E. Holzer (1968), The polar wind, J. Geophys. Res., 73,6846 .

Barakat, A. R., and I. A. Barghouthi (1994), The effect of wave-particle interactions on the polar winds $\mathrm{O}(+)$, Geophys. Res. Lett., 21, 2279.

Barakat, A. R., I. A. Barghouthi, and R. W. Schunk (1995), Doublehump $\mathrm{H}^{+}$velocity distribution in the polar wind, Geophys. Res. Lett., 22, 1857.

Borovsky, J. E., M. F. Thomsen, and D. J. McComas (1997), The superdense plasma sheet: Plasmaspheric origin, solar wind origin, or ionospheric origin?, J. Geophys. Res., 102, 22,089.

Borovsky, J. E., M. Thomsen, and R. C. Elphic (1998), The driving of the plasma sheet by the solar wind, J. Geophys. Res., 103, 17,617.

Cannata, R. W., and T. I. Gombosi (1989), Modeling the solar cycle dependence of quiet-time ion upwelling at high geomagnetic latitudes, Geophys. Res. Lett., 16, 1141.

Capone, L. A., R. C. Whitten, S. S. Prasad, and J. Dubach (1977), The ionospheres of Saturn, Uranus, and Neptune, Astrophys. J., 215, 977.

Cladis, J. B. (1986), Parallel acceleration and transport of ions from polar ionosphere to plasma sheet, Geophys. Res. Lett., 13, 893.

Connerney, J. E. P., and J. H. Waite (1984), New model of saturn's ionosphere with an influx of water from the rings, Nature, 312, 136.

Cowley, S., E. Bunce, and R. Prangé (2004a), Saturn's polar ionospheric flows and their relation to the main auroral oval, Ann. Geophys., 22, 1379

Cowley, S. W. H., E. J. Bunce, and J. M. O’Rourke (2004b), A simple quantitative model of plasma flows and currents in Saturn's polar ionosphere, J. Geophys. Res., 109, A05212, doi:10.1029/2003JA010375.

Cravens, T. E. (1987), Vibrationally excited molecular hydrogen in the upper atmosphere of Jupiter, J. Geophys. Res., 92, 11,083.

Dougherty, M. K., K. K. Khurana, F. M. Neubauer, C. T. Russell, J. Saur, J. S. Leisner, and M. E. Burton (2006), Identification of a dynamic atmosphere at enceladus with the Cassini magnetometer, Science, 311(5766), 1406, doi:10.1126/science.1120985.

Dungey, J. W. (1961), Interplanetary magnetic field and the auroral zones, Phys. Rev. Lett., 6, 47. 
Festou, M. C., and S. K. Atreya (1982), Voyager ultraviolet stellar occultation measurements of the composition and thermal profiles of the saturnian upper atmosphere, Geophys. Res. Lett., 9, 1147.

Feuchtgruber, H., E. Lellouch, T. de Graauw, B. Bezard, T. Encrenaz, and M. Griffin (1997), External supply of oxygen to the atmospheres of giant planets, Nature, 389, 159-162.

Frey, M. A. (1997), The polar wind of Saturn, Ph.D. thesis, Univ. of Mich., Ann Arbor, Mich.

Ganguli, S. B. (1996), The polar wind, Rev. Geophys., 34, 311.

Ganguli, S. B., and P. J. Palmadesso (1987), Plasma transport in the auroral return current region, J. Geophys. Res., 92, 8673.

Ganguli, S. B., H. G. Mitchell, and P. J. Palmadesso (1987), Behavior of ionized plasma in the high latitude topside ionosphere: The polar wind, Planet. Space Sci., 35, 703.

Gardner, L. C., and R. W. Schunk (2005), Global neutral polar wind model, J. Geophys. Res., 110, A10302, doi:10.1029/2005JA011029.

Gombosi, T. I. (1988), Preshock region acceleration of implanted cometary $\mathrm{H}^{+}$and $\mathrm{O}^{+}, J$. Geophys. Res., 93, 35 .

Gombosi, T. I., and K. C. Hansen (2005), Saturn's variable magnetosphere, Science, 307, 1224, doi:10.1126/science.1108226.

Gombosi, T. I., and T. L. Killeen (1987), Effects of thermospheric motions on the polar wind: A time-dependent numerical study, J. Geophys. Res., 92, 4725 .

Gombosi, T. I., and A. Nagy (1989), Time-dependent modeling of field aligned current-generated ion transients in the polar wind, J. Geophys. Res., 94, 359.

Gombosi, T. I., T. E. Cravens, and A. F. Nagy (1985), A time-dependent theoretical model of the polar wind preliminary results, Geophys. Res. Lett., $12,167$.

Gombosi, T. I., L. K. Kerr, A. F. Nagy, and R. W. Cannata (1991), Helium in the polar wind, Adv. Space Res., 12, 183.

Hansen, C. J., L. Esposito, A. I. F. Stewart, J. Colwell, A. Hendrix, W. Pryor, D. Shemansky, and R. West (2006), Enceladus' water vapor plume, Science, 311(5766), 1422, doi:10.1126/science.1121254.

Hansen, K. C., A. J. Ridley, G. B. Hospodarsky, N. Achilleos, M. K. Dougherty, T. I. Gombosi, and G. Tóth (2005), Global MHD simulations of Saturn's magnetosphere at the time of Cassini approach, Geophys. Res. Lett., 32, L20S06, doi:10.1029/2005GL022835.

Hoffman, J. H. (1970), Studies of the composition of the ionosphere with a magnetic deflection mass spectrometer, Int. J. Mass Spectrom. Ion Phys. 4,315 .

Horwitz, J. L., C. W. Ho, H. D. Scarbro, G. R. Wilson, and T. E. Moore (1994), Centrifugal acceleration of the polar wind, J. Geophys. Res., 99, 15,051 .

Huddleston, M. M., C. R. Chappell, D. C. Delcourt, T. E. Moore, B. L. Giles, and M. O. Chandler (2005), An examination of the process and magnitude of ionospheric plasma supply to the magnetosphere, J. Geophys. Res., 110, A12202, doi:10.1029/2004JA010401.

Jackman, C. M., N. Achilleos, E. J. Bunce, S. W. H. Cowley, M. K. Dougherty, G. H. Jones, S. E. Milan, and E. J. Smith (2004), Interplanetary magnetic field at 9 AU during the declining phase of the solar cycle and its implications for Saturn's magnetospheric dynamics, J. Geophys. Res., 109, A11203, doi:10.1029/2004JA010614.

Jensen, M. J., H. B. Pedersen, C. P. Safvan, K. Seiersen, X. Urbain, and L. H. Andersen (2001), Dissociative recombination and excitation of $h_{3}^{+}$, Phys. Rev. A, 63(5), 052701.

Jurac, S., and J. D. Richardson (2005), A self-consistent model of plasma and neutrals at Saturn: Neutral cloud morphology, J. Geophys. Res., 110, A09220, doi:10.1029/2004JA010635.

Kim, Y. H., and J. L. Fox (1994), The chemistry of hydrocarbon ions in the Jovian ionosphere, Icarus, 112, 310, doi:10.1006/icar.1994.1186.

Majeed, T., and J. C. McConnell (1991), The upper ionospheres of jupiter and saturn, Planet. Space Sci., 39, 1715.

Majeed, T., R. V. Yelle, and J. C. McConnell (1991), Vibrationally excited h2 in the outer planets thermosphere: Fluorescence in the lyman and werner bands, Planet. Space Sci., 39, 1591, doi:10.1016/00320633(91)90085-O.

McElroy, M. B. (1973), The ionospheres of the major planets, Space Sci. Rev., 14, 460.

Michael, M., R. E. Johnson, F. Leblanc, M. Liu, J. G. Luhmann, and V. I. Shematovich (2005), Ejection of nitrogen from Titan's atmosphere by magnetospheric ions and pick-up ions, Icarus, 175, 263, doi:10.1016/ j.icarus.2004.11.004.

Mitchell, H. G., and P. J. Palmadesso (1983), A dynamic model for the auroral field line plasma in the presence of field-aligned current, J. Geophys. Res., 88, 2131.
Moore, L., and M. Mendillo (2005), Ionospheric contribution to Saturn's inner plasmasphere, J. Geophys. Res., 110, A05310, doi:10.1029/ 2004JA010889.

Moore, L. E., M. Mendillo, I. C. F. Müller-Wodarg, and D. L. Murr (2004), Modeling of global variations and ring shadowing in Saturn's ionosphere, Icarus, 172, 503, doi:10.1016/j.icarus.2004.07.007.

Moore, T. E., and D. C. Delcourt (1995), The geopause, Rev. Geophys, 33, 175 .

Moses, J. I., and S. F. Bass (2000), The effects of external material on the chemistry and structure of Saturn's ionosphere, J. Geophys. Res., 105, 7013

Nagy, A. F. (1987), Photochemistry of planetary ionospheres, Adv. Space Res., 7, 89.

Nagy, A. F., A. R. Barakat, and R. W. Schunk (1986), Is Jupiter's ionosphere a significant plasma source for its magnetosphere?, J. Geophys. Res., 91, 351.

Nagy, A. F., et al. (2005), Recent results of ionospheric occultations of the Cassini spacecraft by Saturn, in AAS/Division for Planetary Sciences Meeting Abstracts, vol. 37, pp. 83-84, Cambridge Univ. Press, New York.

Pierrard, V., and J. Lemaire (1998), A collisional kinetic model of the polar wind, J. Geophys. Res., 103, 11,701.

Porco, C. C., et al. (2006), Cassini observes the active south pole of Enceladus, Science, 311(5766), 1393, doi:10.1126/science.1123013.

Richardson, J. D., and S. Jurac (2004), A self-consistent model of plasma and neutrals at Saturn: The ion tori, Geophys. Res. Lett., 31, L24803, doi:10.1029/2004GL020959.

Schunk, R. W. (1981), Electron temperature anisotropy in the polar wind, J. Geophys. Res., 86, 91.

Schunk, R. W., and A. F. Nagy (2000), Ionospheres: Physics, Plasma Physics, and Chemistry, Cambridge Univ. Press, New York.

Schunk, R. W., and J. J. Sojka (1989), A three-dimensional time-dependent model of the polar wind, J. Geophys. Res., 94, 8973.

Sharp, R. D., W. Lennartsson, and R. J. Strangeway (1985), The ionospheric contribution to the plasma environment in near-Earth space, Radio Sci., 20, 456.

Shemansky, D. E., J. T. Hallett, and X. Liu (2005), The first Cassini UVIS stellar occultation of the Saturn atmosphere, Eos Trans. AGU, volume?, Fall Meet. Suppl., Abstract?

Shematovich, V. I., R. E. Johnson, M. Michael, and J. G. Luhmann (2003), Nitrogen loss from Titan, J. Geophys. Res., 108(E8), 5087, doi:10.1029/ 2003JE002094

Smith, G. R., D. E. Shemansky, J. B. Holberg, A. L. Broadfoot, B. R. Sandel, and J. C. McConnell (1983), Saturn's upper atmosphere from the Voyager 2 EUV solar and stellar occultations, J. Geophys. Res., 88, 8667.

Smith, H. T., R. E. Johnson, and V. I. Shematovich (2004), Titan's atomic and molecular nitrogen tori, Geophys. Res. Lett., 31, L16804, doi:10.1029/2004GL020580.

Smith, H. T., M. Shappirio, E. C. Sittler, D. Reisenfeld, R. E. Johnson, R. A. Baragiola, F. J. Crary, D. J. McComas, and D. T. Young (2005), Discovery of nitrogen in Saturn's inner magnetosphere, Geophys. Res. Lett., 32, L14S03, doi:10.1029/2005GL022654.

Spencer, J. R., et al. (2006), Cassini encounters Enceladus: Background and the discovery of a south polar hot spot, Science, 311(5766), 1401, doi:10.1126/science. 1121661 .

Tóth, G., et al. (2005), Space Weather Modeling Framework: A new tool for the space science community, J. Geophys. Res., 110(A12), A12226, doi:10.1029/2005JA011126.

VanLeer, B. (1979), Towards the ultimate conservative difference scheme. V. A second order sequel to Godunov's method, J. Comput. Phys., 32, 101

Waite, J., et al. (2006), Cassini Ion and Neutral Mass Spectrometer: Enceladus plume composition and structure, Science, 311(5766), 1419, doi:10.1126/science.1121290.

Winglee, R. M. (2000), Mapping of ionospheric outflows into the magnetosphere for varying IMF conditions, J. Atmos. Terr. Phys., 62, 527.

Young, D. T., et al. (2005), Composition and dynamics of plasma in Saturn's magnetosphere, Science, 307, 1262, doi:10.1126/science.1106151.

A. Glocer, T. Gombosi, K. C. Hansen, A. Nagy, A. J. Ridley, and G. Toth, Center for Space Environment Modeling, University of Michigan, Ann Arbor, MI, 48109-2143, USA. (aglocer@umich.edu; tamas@umich. edu; kenhan@umich.edu; anagy@umich.edu; ridley@umich.edu; gtoth@ umich.edu) 